\title{
Planetary Dynamos from a Solar Perspective
}

\author{
U.R. Christensen · D. Schmitt • M. Rempel
}

Received: 1 July 2008 / Accepted: 30 September 2008 / Published online: 16 October 2008

(C) Springer Science+Business Media B.V. 2008

\begin{abstract}
Direct numerical simulations of the geodynamo and other planetary dynamos have been successful in reproducing the observed magnetic fields. We first give an overview on the fundamental properties of planetary magnetism. We review the concepts and main results of planetary dynamo modeling, contrasting them with the solar dynamo. In planetary dynamos the density stratification plays no major role and the magnetic Reynolds number is low enough to allow a direct simulation of the magnetic induction process using microscopic values of the magnetic diffusivity. The small-scale turbulence of the flow cannot be resolved and is suppressed by assuming a viscosity far in excess of the microscopic value. Systematic parameter studies lead to scaling laws for the magnetic field strength or the flow velocity that are independent of viscosity, indicating that the models are in the same dynamical regime as the flow in planetary cores. Helical flow in convection columns that are aligned with the rotation axis play an important role for magnetic field generation and forms the basis for a macroscopic $\alpha$-effect. Depending on the importance of inertial forces relative to rotational forces, either dynamos with a dominant axial dipole or with a small-scale multipolar magnetic field are found. Earth is predicted to lie close to the transition point between both classes, which may explain why the dipole undergoes reversals. Some models fit the properties of the geomagnetic field in terms of spatial power spectra, magnetic field morphology and details of the reversal behavior remarkably well. Magnetic field strength in the dipolar dynamo regime is controlled by the available power and found to be independent of rotation rate. Predictions for the dipole moment agree well with the observed field strength of Earth and Jupiter and moderately well for other planets. Dedicated dynamo models for Mercury, Saturn, Uranus and Neptune, which assume stably stratified layers above or below the dynamo region, can explain some of the unusual field properties of these planets.
\end{abstract}

Keywords Planetary magnetic fields · Geodynamo $\cdot$ Dynamo models

U.R. Christensen (凶) • D. Schmitt

Max-Planck-Institut für Sonnensystemforschung, 37191 Katlenburg-Lindau, Germany

e-mail: christensen@mps.mpg.de

M. Rempel

High Altitude Observatory, National Center for Atmospheric Research, Boulder, CO 80307-3000, USA 


\section{Introduction}

Starting in 1995 numerical modeling of the Earth's dynamo has flourished with remarkable success. Direct numerical simulation of convection-driven MHD-flow in a rotating spherical shell show magnetic fields that resemble the geomagnetic field in many respects: they are dominated by the axial dipole of approximately the right strength, they show spatial power spectra similar to that of Earth, and the magnetic field morphology and the temporal variation of the field resembles that of the geomagnetic field (Christensen and Wicht 2007). Some models show stochastic dipole reversals whose details agree with what has been inferred from paleomagnetic data (Glatzmaier and Roberts 1995; Kutzner and Christensen 2002; Wicht 2005). While these models represent direct numerical simulations of the fundamental MHD equations without parameterized induction effects, they do not match actual planetary conditions in a number of respects. Specifically, they rotate too slowly, are much less turbulent, and use a viscosity and thermal diffusivity that is far too large in comparison to magnetic diffusivity. Because of these discrepancies, the success of geodynamo models may seem surprising.

In order to better understand the extent to which the models are applicable to planetary dynamos, scaling laws that relate basic properties of the dynamo to the fundamental control parameters play an important role. In recent years first attempts have been made to derive such scaling laws from a set of numerical simulations that span the accessible parameter space (Christensen and Tilgner 2004; Christensen and Aubert 2006). The extrapolation of these laws to planetary parameters gives reasonable results, which suggests that despite their shortcomings the dynamo models are already in the appropriate dynamical regime.

Most planets in the solar system have internal magnetic fields or once had such fields (Stevenson 2003). In many, but not all, cases the axial dipole dominates the field at the planetary surface. In fact, a surprising diversity is found in magnetic field strength and field morphology. A comparative dynamo theory, that explains the common features and the differences between planetary magnetic fields, is still in its infancy.

This paper intends to give a brief overview on the progress made in understanding planetary dynamos, mostly achieved with the aid of numerical simulations. We will first review the salient properties of the geomagnetic field and then proceed to what is known on magnetism of other planets. Next we present the conceptual assumptions used in modeling planetary dynamos, and we discuss some essential differences to the solar dynamo. We then describe successes and failures in modeling the geodynamo and proceed to discuss attempts to derive scaling laws that put the understanding on a more general level. We close by discussing some hypotheses and models to explain the observed magnetic properties of specific planets other than Earth.

\section{Planetary Magnetic Fields}

\subsection{Geomagnetic Field}

The properties of the recent geomagnetic field have been mapped with high spatial resolution by dedicated satellite missions in a low Earth orbit, such as MAGSAT, ØRSTED, and CHAMP (Olsen et al. 2007). The variation of the field during the last four centuries (the so-called historical field) has been recorded with somewhat lower spatial resolution by a network of magnetic observatories and by individual measurements, most of which have been performed routinely by mariners (Jackson et al. 2000). Going further back in time, information on the geomagnetic field has been retrieved from the remanent magnetization in 
man-made artifacts, covering the last couple of thousand years (Korte and Constable 2005), or in natural rocks, covering geological time as far back as 3 billion years (Tarduno et al. 2007). The spatial and temporal resolution is moderate for archeomagnetic data and poor for paleomagnetic data.

Historically, a variety of hypotheses have been proposed for the origin of the geomagnetic field. The only viable model that survived the test of time is that of a dynamo process operating in the Earth's core. Seismology and high pressure research have shown that its outer part is an iron-rich metallic liquid, which contains approximately $10 \%$ of light alloying elements (such as sulphur, oxygen or silicon in unknown proportions). There is a small solid inner core that is depleted in the light elements relative to the outer core. Secular cooling of the core implies that the inner core grows by freezing iron on its surface, enriching the overlying residual liquid in the light elements. Both thermal and compositional convection drive a circulation in the outer core. Hence the most basic requirement for a dynamo, flow of an electrically conducting fluid, seems to be satisfied inside the Earth.

While the geomagnetic field is observed at or above the Earth's surface, maps of the field at the top of the iron core are more useful for interpreting field structure in terms of the underlying dynamo process. For the historical geomagnetic field such maps have been constructed under the assumption of a potential field outside the core (Gubbins and Bloxham 1985; Jackson et al. 2000). This assumption seems to be satisfied approximately for the long-wavelength part of the magnetic field up to spherical harmonic degree 13. At smaller scales the field at the Earth's surface is dominated by contributions arising from the inhomogeneous remanent magnetization of rocks in the Earth's crust. Therefore, it is not possible to determine the structure of the core field at wavelengths smaller than $2000 \mathrm{~km}$.

Figure 1a shows the radial component of Earth's field at the core-mantle boundary. The axial dipole component is dominant, but higher multipoles contribute to the overall field morphology more strongly than they do at the Earth's surface. In particular, the magnetic flux contributing most prominently to the axial dipole field is concentrated in two lobes in each hemisphere at approximately $60^{\circ}-70^{\circ}$ latitude. The flux close to the rotation poles is weak or slightly inverse with respect to the dipole polarity. At low latitudes flux spots of both polarities exist.

The rms field strength at the top of Earth's core is approximately $0.4 \mathrm{mT}$ (4 Gauss) in degrees up to 13. It is plausible to assume that the magnetic field $B$ inside the core is larger by a factor between three and ten, which puts the characteristic field strength in the dynamo region into the range between one and a few mT. This means that the Elsasser number $\Lambda$

$$
\Lambda=\frac{B^{2}}{\mu_{o} \eta \rho \Omega}
$$

is of order one, where $\mu_{o}$ is magnetic permeability, $\eta$ magnetic diffusivity, $\rho$ density and $\Omega$ rotation rate. The Elsasser number is often taken as measure for the ratio of Lorentz forces to Coriolis forces acting on the flow. The finding that $\Lambda \approx 1$ in the Earth's core has been taken as support for the notion that planetary dynamos are in a magnetostrophic regime, where Coriolis force and Lorentz force balance to first order, and that this balance controls the strength of the magnetic field (e.g., Stevenson 2003). As we will see in Sect. 8, dynamo simulations put some doubt on this hypothesis.

The Earth's magnetic field changes significantly on time scales of one hundred years. This secular variation concerns mainly the higher multipole components. The axial dipole component is much more stable, at least in terms of polarity. Polarity changes occur stochastically in time intervals of some hundred thousand years. The duration of a reversal 
Fig. 1 a Radial component of the geomagnetic field at the core-mantle boundary in 1990 , red colors for inward field, blue colors for outward field.

b Snapshot of radial field from a dynamo model with parameters $E=10^{-5}, R a^{*}=0.12$,

$P m=0.8, P r=1$. c Same snapshot for the field low-pass filtered to spherical harmonic degrees $<14$
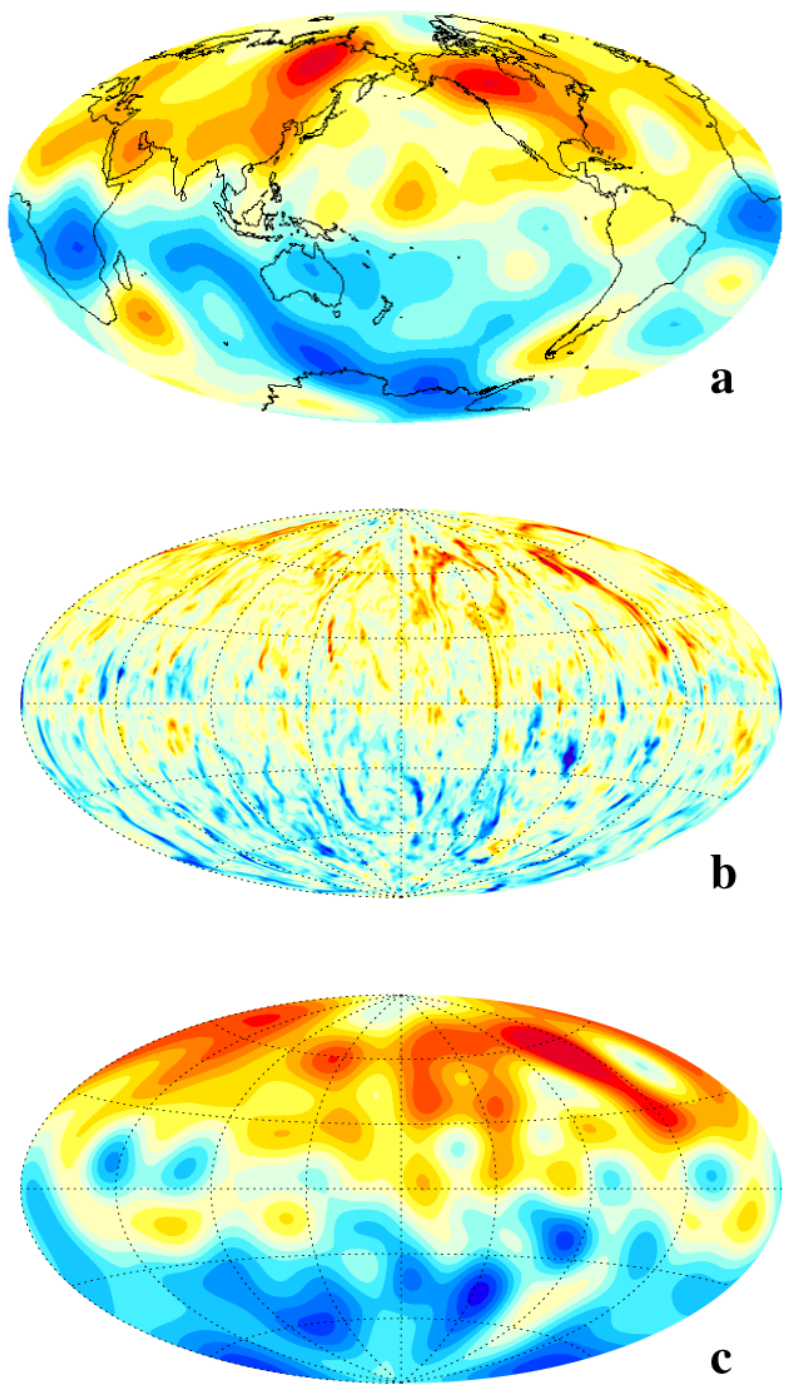

is comparatively short, on the order of a few thousand years. The frequency of reversals itself varies on time scales of 100 million years, which is the typical time scale for mantle convection. This is interpreted as reflecting the coupling of the dynamo to heterogeneities (for example in temperature) in the Earth's lower mantle (Glatzmaier et al. 1999; Kutzner and Christensen 2004; Courtillot and Olson 2007).

\subsection{Other Planets}

The magnetic fields of all major planets in the solar system have been characterized by space missions, although often this provided only a crude snapshot compared to our knowledge of the geomagnetic field. In particular, next to nothing is known about the time variability of other planetary fields. Table 1 gives an overview on the field properties. 
Table 1 Planetary magnetic fields

\begin{tabular}{llllrl}
\hline Planet & Active dynamo & Past dynamo & $r_{c} / r_{p}$ & $B_{S}[\mathrm{nT}]$ & Structure \\
\hline Mercury & Yes ? & & 0.75 & 400 & dipolar \\
Venus & No & $?$ & 0.55 & & dipolar \\
Earth & Yes & Yes & 0.55 & 50,000 & \\
Moon & No & Yes ? & $0.2 ?$ & & dipolar \\
Mars & No & Yes & 0.5 & & dipolar \\
Jupiter & Yes & & 0.85 & 500,000 & dipolar \\
Ganymede & Yes & & $0.3 ?$ & 1,000 & multipolar \\
Saturn & Yes & & 0.5 & 30,000 & multipolar \\
Uranus & Yes & & 0.7 & 45,000 & 45,000 \\
Neptune & Yes & & 0.7 & & \\
\hline
\end{tabular}

Probably all large planetary bodies have an electrically conducting fluid core (or, at least had a fluid core at some time in their history) that can potentially sustain a dynamo. However, the nature of this core region is quite different between the various groups of planets. In the terrestrial planets it consists of a molten iron-rich alloy. In Jupiter and Saturn it is a metallic high-pressure form of hydrogen and the deep interior of Uranus and Neptune consists of a water-rich fluid with ionic conductivity. In Jupiter's satellite Ganymede, which has a magnetic field of its own, the dynamo probably resides in a deep liquid iron core, although dynamo action in a salty water ocean at shallower depth can perhaps not be excluded entirely. The values of the core radius $r_{c}$ relative to the planetary radius $r_{p}$ are given in Table 1.

Not all large planetary bodies have a magnetic field at present, although in some cases (Moon, Mars) local magnetic fields arise from remanent magnetization of minerals in the planet's crust (akin to so-called magnetic anomalies at Earth). The most likely explanation of how the magnetization was acquired is that an internal dynamo generated a strong magnetic field early in the planet's history.

In most cases the magnetic field at the planetary surface is dominated by a dipole that is nearly aligned with the rotation axis. As in case of the Earth higher multipoles contribute on the order of $10 \%$ to the fields at the surface of Jupiter and Saturn. Mercury's field morphology has not yet been characterized sufficiently to clearly quantify the non-dipole contributions. A particular property of Saturn's magnetic field not found at other planets is an extremely high degree of axisymmetry including a dipole tilt relative to the rotation axis that is indistinguishable from zero. In the case of Uranus and Neptune, the quadrupole and octupole contribute to the surface field at a similar level as the dipole and the latter is strongly tilted against the rotation axis. When continued downward to radius $r_{c}$ the dipole still remains the dominant field component in most planets, but becomes relatively weaker than higher multipoles in the cases of Uranus and Neptune. Therefore, the field of the latter two planets is characterized as 'multipolar', in Table 1.

The magnetic field strength at the planetary surface, $B_{s}$, covers a wide range for the different planets, from ten times the strength of the geomagnetic field at Jupiter to $1 \%$ of the Earth's field strength in the case of Mercury. When downward continuing the magnetic field to $r_{c}$ the differences in field strength are slightly reduced but remain large between the end members.

In summary, despite some similarities, large differences in field strength and structure exist. Theory must strive at explaining them either in terms of particular conditions in the 
planet, on a case-by-case basis, or by establishing laws describing a systematic dependence of the dynamo properties on the essential controlling parameters. Which these parameters are is not entirely clear a-priori, but size, rotation rate, electrical conductivity and vigor of convection are some obvious candidates. To explain the diversity of magnetism in the planetary system, a combination of these two approaches seems to be necessary.

\section{Solar Versus Planetary Dynamos}

In contrast to the dipole-dominated geomagnetic field, which undergoes stochastic reversals, the solar magnetic field is dominated by small scales, yet it shows a high degree of regularity. This is expressed in the eleven-year activity cycle, the systematic latitudinal migration of the emergence region of sunspots and the east-west alignment of bipolar active regions that follow Hale's polarity rules. These differences in magnetic field behavior point at profound differences in the underlying dynamo process. In this section we discuss some essential differences between the solar dynamo and planetary dynamos and their implications for the modeling of planetary dynamos.

\subsection{Energetics}

While strong convection is a matter of fact in the outer parts of the Sun, the occurrence of sufficient flow that can drive a dynamo is less clear for planetary cores, in particular in the case of the terrestrial planets. The energy flux in planets is obviously much weaker and results from the slow secular cooling that releases internal energy (of gravitational origin) acquired during accretion. This may be augmented by heat due to the decay of radioactive trace elements, although it is uncertain if the iron cores contain significant amounts of them (e.g. Rama Murthy et al. 2003). While radiative heat transport plays no significant role in planetary dynamo regions, a substantial fraction of the heat flow in the iron cores of terrestrial planets can be transported by conduction along an adiabatic temperature gradient. It is important to note that the heat flow from the core into the overlying mantle made of solid silicate rock is controlled by the very sluggish convective circulation in the mantle (the core delivers as much heat as the mantle is able to carry away). Most models that consider the energy budget of the Earth's interior conclude that the heat flow at the top of the core is larger than the 'adiabatic heat flow' (Nimmo 2007), although a slightly subadiabatic heat flow cannot be excluded. The latter case implies a stable thermal stratification in the upper part of the liquid core, which may be overcome in the Earth by compositional convection arising from inner core growth. Also, the latent heat of inner core solidification is an important effective heat source that ensures a superadiabatic heat flow at greater depth in the core. The lack of plate tectonics on planets other than Earth means less efficient heat loss, implying slower cooling of the core. The heat flow at the core-mantle boundary is probably subadiabatic in these planets. Furthermore, if the planet has failed to nucleate a solid inner core, compositional buoyancy is unavailable, and the fluid core would not convect at all. This may be the case in Mars and Venus and would explain the absence of a present dynamo. Early in the planet's history cooling rates have probably been higher. In the case of Mars, a thermally driven dynamo that has operated in the first 500 million years would explain the strong magnetization of parts of the old Martian highland crust which has been formed at this time. 


\subsection{Magnetic Turbulence}

The magnetic Reynolds number

$$
R m=\frac{U D}{\eta},
$$

where $U$ is the characteristic flow velocity, $D$ the thickness of the convecting layer and $\eta$ the magnetic diffusivity, is very different for stellar and planetary dynamos. For the solar dynamo it is of the order $10^{10}$ using the microscopic value of magnetic diffusivity deep in the convection zone. The induction effects of the highly turbulent flow and magnetic field cannot be captured in direct global simulations of the solar dynamo, and is often treated in parameterized form in the framework of the mean-field dynamo concept (Krause and Rädler 1980); for recent reviews see Ossendrijver (2003) and Charbonneau (2005).

For the Earth's core, $R m$ can be estimated to be of the order 1000 (Christensen and Tilgner 2004) and in the dynamo regions of the hydrogen planets it is probably an order of magnitude larger (Stevenson 2003). At least for the geodynamo and for dynamos of other terrestrial planets the value of the magnetic Reynolds number is sufficiently small to allow a direct numerical solution of the magnetic induction equation in global models, without the need to introduce parameterizations for the magnetic induction, such as an $\alpha$-effect or an effective magnetic diffusivity. The ability of running the simulations at the correct value of the magnetic Reynolds number is perhaps the essential reason for the success of geodynamo models. The hydrodynamic Reynolds number is of order $10^{8}$ in planetary dynamos and therefore far too large to capture the small-scale turbulence of the flow in a direct simulation. These flow scales may be too small to have a direct effect on the magnetic field, however, by their back-reaction on the large scale-flow they could play a role for the dynamo.

\subsection{Stratification}

The density in the Sun varies by many orders of magnitude and the convection region spans many density scale heights. The dynamo region in planets covers roughly one scale height in Jupiter and substantially less in all other planets. It is not possible to simulate the entire solar convection zone in one comprehensive model. Large scale simulations typically neglect the upper most $5-10 \%$ of the solar convection zone where the pressure scale height is small and where the sound velocity is not much larger than the flow velocity and the anelastic approximation breaks down (Miesch 2005). The strong density changes are thought to play an important role for the solar dynamo. Coherent flow helicity, which is an essential ingredient for the dynamo process, arises in the Sun because of the action of the Coriolis force on rising expanding and sinking contracting parcels of plasma. Strong magnetic flux tubes have their own dynamics, because the reduction of fluid pressure that compensates magnetic pressure makes them buoyant. Both effects probably do not play a significant role in planetary dynamos; therefore most present geodynamo models neglect the small density variation and assume incompressible flow in the Boussinesq approximation. In the incompressible case, other mechanisms generate helicity that is preferentially negative in the Northern hemisphere and positive in the southern hemisphere (see Olson et al. 1999, for a discussion).

Of particular importance for the solar dynamo is the tachocline between the convection zone and the radiative interior of the Sun (Hughes et al. 2007), where differential rotation generates a strong toroidal magnetic field and the subadiabatic stratification ensures its storage for a sufficiently long time, until magnetic buoyancy leads to the rise of magnetic flux through the convection zone and emergence at the photosphere (Caligari et al. 1995; Fan 2004). For planetary dynamos it is usually assumed that the process of magnetic field generation occurs in the bulk of the convecting layer. 


\subsection{Inertial Forces}

Another difference between the solar dynamo and planetary dynamos is the potential role of inertial forces. Their importance relative to the Coriolis force is measured by the Rossby number

$$
R o=\frac{U}{\Omega L},
$$

where $U$ and $L$ are characteristic velocity and length scale, respectively, and $\Omega$ is the rotation frequency. In the solar convection zone $R o \approx 1$. Using estimates for the flow velocity at the top of the Earth's core of the order $1 \mathrm{~mm} / \mathrm{sec}$ from the secular variation of geomagnetic field, the Rossby number is of order $10^{-6}$ when a global scale such as the core radius or $D$ is used for $L$. Therefore, fluid motion in the Earth is often considered to be largely unaffected by inertial forces (except for special modes of motion termed 'torsional oscillations', representing collective motion on cylinders that are coaxial to the rotation axis). The general force balance is believed to be that between Coriolis force, pressure gradient force, Lorentz forces and buoyancy forces (magnetostrophic balance; Roberts 1987). However, inertial forces may become important at small length scales and can potentially feed back on the large scale flow (see Sect. 5).

\section{Setup and Parameters for Geodynamo Models}

For the large-scale solar dynamo direct numerical simulations of the anelastic magnetohydodynamic equations have been successful in demonstrating self-excited dynamo action and in reproducing the internal differential rotation of the Sun (e.g. Miesch 2005). But so far they fail to reproduce the cyclic behavior and the latitudinal propagation of the solar magnetic activity (Brun et al. 2004), despite the fact that most of these solar models are comparable to geodynamo models in terms of their resolution and the degree of turbulence reached. This may indicate that in the case of the Sun important induction effects occur at scales below the currently achievable resolution or in boundary layers, such as the tachocline. Currently these effects can only be modeled by local 3-D simulations or in global mean-field models. In contrast, self-consistent global simulations are able to reproduce the observed first-order properties of the geomagnetic field and much of our understanding of planetary dynamos is now based on the results of such simulations.

Most geodynamo models use basically the same setup. The equations for convective flow in the Boussinesq limit and the magnetic induction equation are solved for a rotating and electrically conducting spherical shell. Usually non-dimensional variables and dimensionless control parameters are employed. As an example, we give the equations in the form used by Christensen and Aubert (2006):

$$
\begin{gathered}
\frac{\partial \mathbf{u}}{\partial t}+\mathbf{u} \cdot \nabla \mathbf{u}+2 \hat{\mathbf{z}} \times \mathbf{u}+\nabla \Pi=E \nabla^{2} \mathbf{u}+R a^{*} \frac{\mathbf{r}}{r_{o}} T+(\nabla \times \mathbf{B}) \times \mathbf{B}, \\
\frac{\partial \mathbf{B}}{\partial t}-\nabla \times(\mathbf{u} \times \mathbf{B})=\frac{E}{P m} \nabla^{2} \mathbf{B}, \\
\frac{\partial T}{\partial t}+\mathbf{u} \cdot \nabla T=\frac{E}{P r} \nabla^{2} T, \\
\nabla \cdot \mathbf{u}=0, \quad \nabla \cdot \mathbf{B}=0 .
\end{gathered}
$$


$\mathbf{u}$ is velocity, $\Pi$ dynamical pressure, $T$ temperature, $\mathbf{B}$ magnetic induction, and the unit vector $\hat{\mathbf{z}}$ indicates the direction of the rotation axis. The four non-dimensional control parameters are the Ekman number

$$
E=\frac{v}{\Omega D^{2}},
$$

a modified Rayleigh number

$$
R a^{*}=\frac{\alpha g_{o} \Delta T}{\Omega^{2} D},
$$

the Prandtl number

$$
\operatorname{Pr}=\frac{v}{\kappa},
$$

and the magnetic Prandtl number

$$
P m=\frac{v}{\eta}
$$

where $v$ is viscosity, $\alpha$ the thermal expansion coefficient, $g_{o}$ gravity at the outer boundary, $\Delta T$ the (superadiabatic) temperature contrast, and $\kappa$ the thermal diffusivity. We note that $\left(R a^{*}\right)^{1 / 2}$ is often called the convected Rossby number in the astrophysical literature. $R a^{*}$ is related to the conventional Rayleigh number $R a$ by $R a^{*}=R a E^{2} P^{-1}$.

In Table 2 we compare control parameter values used in geodynamo models with those for the Earth's core. The Rayleigh number has been normalized with its critical value $R a_{c}^{*}$ for the onset of convection in the absence of a magnetic field. We also list several other non-dimensional numbers that characterize the dynamo and that are a result of the model solution. The magnetic Reynolds number $R m$ agrees with Earth values at least in the more advanced models, whereas the hydrodynamic Reynolds number $R e=U D / v$ is far too small and the Rossby number $R o$ is too large. The Elsasser number $\Lambda$ can be taken as a nondimensional measure for the magnetic field strength. The claim that the models reproduce the geomagnetic field strength actually means that they give an Elsasser number of order one.

While the Prandtl number in the models is of the right order, the values of the other control parameters are far off. The Ekman number and the magnetic Prandtl number are too large by factors of $10^{10}$ and $10^{6}$, respectively. The modified Rayleigh number is too small with respect to supercriticality, but, as we will later see, its absolute value is larger than the core value. In terms of physical parameters, the viscosity (and thermal diffusivity)

Table 2 Order of magnitude of parameters in the core and in dynamo models

\begin{tabular}{lllll}
\hline & \multicolumn{2}{l}{ Control Parameters } & & \\
\cline { 2 - 5 } & $R a^{*} / R a_{c}^{*}$ & $E$ & $10^{-6}-10^{-5}$ & \\
\hline Earth's core & $\approx 5000$ & $10^{-15}-10^{-14}$ & $0.1-10^{3}$ & $0.1-1$ \\
Models & $1-100$ & $10^{-3}-10^{-6}$ & & $0.1-10^{3}$ \\
\hline \multirow{2}{*}{ Diagnostic numbers } & $R e$ & $\Lambda$ \\
\cline { 2 - 5 } & $R m$ & $10^{8}-10^{9}$ & $\approx 10^{-6}$ & $0.1-10$ \\
Earth's core & $\approx 10^{3}$ & $<2000$ & $3 \times 10^{-4}-10^{-2}$ & $0.1-100$ \\
Models & $50-10^{3}$ & & &
\end{tabular}


is too large by a factor of order $10^{6}$ (compared to the magnetic diffusivity, which is about right). In addition, the rotation rate is too small by a factor of $\approx 10^{4}$ in most models. An exception are models by Glatzmaier and Roberts $(1996,1997)$, who use the right rotation rate at the expense of an even larger viscosity and of values for the two Prandtl numbers much in excess of one. Because the commonly employed codes work most efficiently when the Prandtl numbers are of order one, most modelers preferred the former choice. Perhaps somewhat surprisingly, the two types of models do not show fundamental differences in their magnetic field structure.

Most planetary dynamo models use a spectral transform technique, in which all variables are expanded in spherical harmonic functions concerning the angular dependence. In the radial direction different schemes are used, for example an expansion in Chebychev polynomials combined with a collocation method or a finite difference representation. Linear terms in the governing equations, for which the spherical harmonic modes decouple, are treated implicitly. Non-linear terms (often also the Coriolis force term) are treated explicitly and are evaluated on a spatial grid, which requires back-and-forth transformations at each time step. A detailed account of the standard method is given in Christensen and Wicht (2007).

\section{Classes of Dynamo Solutions}

Many published geodynamo models show a strong dipole field (an example is shown in Fig. 1b, c). Often the dipole in such models shows no tendency to ever reverse, although the model run time may not have been long enough to capture one of these rare events. Some dynamos show a quadrupole-dominated field, or an exotic geometry with a strong field in only one hemisphere (Grote et al. 2000; Grote and Busse 2000; Simitev and Busse 2005). In many of the non-dipolar dynamos the magnetic field structure is spatially complex (Fig. 2) and changes rapidly with time. The spatial power spectrum at the outer boundary of the dynamo models is typically white, but the dipole stands above the higher multipoles in one class of solutions and falls below the multipole level in the other class (Fig. 3). In the fully developed multipolar regime the weak dipole component changes its polarity continuously in an erratic way.

Kutzner and Christensen (2002) found that a transition from dipolar to multipolar dynamos occurs when the convective driving is enhanced. Comparing a set of models in which the relative importance of the inertial force was varied Sreenivasan and Jones (2006) found that inertia plays an important role for the selection of the dynamo regime. Christensen and Aubert (2006) analyzed a large number of model cases, varying all four control parameters. They determined that a transition from dipolar to multipolar magnetic field occurs when a local Rossby number $R o_{\ell}$, in which a characteristic flow length scale $\ell$ determined from the kinetic energy spectrum is used for $L$ in (3), exceeds a critical value of approximately 0.12 . Arguably $R o_{\ell}$ is a more accurate measure for the ratio between inertial force and Coriolis force than the Rossby number Ro formed with the global length scale. Olson and Christensen (2006) confirmed the rule of the local Rossby number as selection criterion for the dynamo regime by including additional dynamo solutions from the literature into the analysis. They also found that dipolar dynamos that do show occasional reversals have a local Rossby number that is near the transitional value. Hence a reversal may represent an accidental brief lapse of the basically dipolar dynamo into the multipolar regime. In fact, during a model reversal the magnetic power spectrum (blue band in Fig. 3) resembles the spectrum of a (permanently) multipolar dynamo. When the dipole recovers it may then take either polarity. 
Fig. 2 Snapshot of radial field from a dynamo model with a multipolar surface field.

Parameters are $E=10^{-5}$ $R a^{*}=0.17, P m=0.5, \operatorname{Pr}=1$. Top panel at full resolution and in bottom panel at degrees 1-14
Planetary dynamos
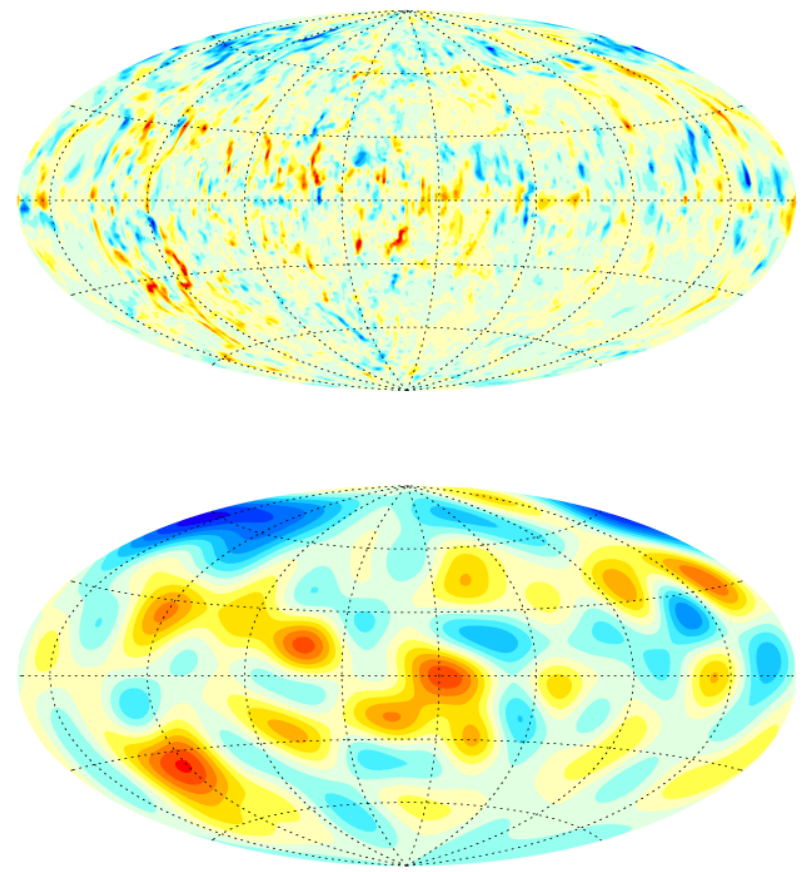

Olson and Christensen (2006) derived an empirical rule based on the model data set of Christensen and Aubert (2006) for relating the local Rossby number to the fundamental control parameters of the dynamo. It involves powers of all four control parameters and obviously requires an extrapolation over a large range to apply it to the planets. Nonetheless, using appropriate parameter values for the Earth, a value of $R o_{\ell} \approx 0.1$ is predicted for the geodynamo, which puts it close to the transition point between the dipolar and the multipolar class (see also Sect. 8 for the application to other planets). A problem is that the predicted

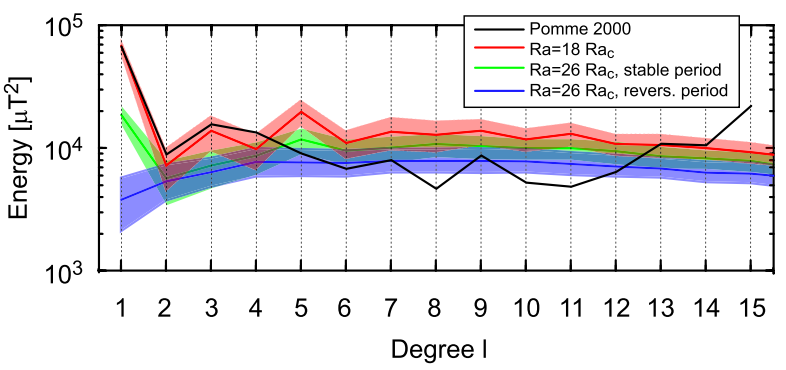

Fig. 3 Spatial power spectra at the outer boundary of the dynamo. Black: Spectrum at Earth's core-mantle boundary from surface observations (Maus et al. 2006), red: spectrum of a dynamo model in the dipolar regime. Spectra of a model in the reversing regime are shown during periods of stable dipole polarity (green) and during reversals (blue). In the model cases the lines represent time-averages and the bands indicate standard deviation. Model parameters are $E=3 \times 10^{-4}, P r=1, P m=3, R a^{*} / R a_{c}^{*}=18$ (red) and 26 (green and blue) 
characteristic flow length scale associated with this value of $R o_{\ell}$ is only of the order $100 \mathrm{~m}$ in the Earth's core. At these scales the magnetic field is diffusion-dominated and cannot be affected directly by the flow. However, in rotational flow an inverse cascade can transport energy through the action of Reynolds stresses to larger scales (e.g. Christensen 2002) which are relevant for the magnetic induction process. Hence the inertial effects at small flow scales may affect the magnetic field structure in an indirect way.

\section{Flow Structure and Field Generation Mechanism}

For the solar dynamo the stretching of magnetic field lines by differential rotation, particularly at the tachocline, is thought to be of major importance for the generation of a toroidal magnetic fields that is much stronger than the poloidal field. The regeneration of the poloidal component of the magnetic field is thought to be by helical fluid motions ( $\alpha$-effect), but the details of the physical mechanism and its precise location have not yet been identified with confidence (Charbonneau 2005). In most geodynamo models differential rotation is not a dominant part of the flow field and the toroidal and poloidal magnetic field components have similar strength (an exceptions are the models by Kuang and Bloxham 1997, 1999). The flow is strongly organized by rotational forces and is fundamentally different in the regions inside and outside the tangent cylinder (an imaginary cylinder that is co-axial with the direction of rotation $\hat{z}$ and touches the inner core at the equator). The most prominent flow pattern are convection columns outside the tangent cylinder that align with the rotation axis. The main circulation is nearly geostrophic, i.e. nearly perpendicular to and independent of $z$. Superimposed is an ageostrophic flow along the column axis that converges at the equator in columns with a cyclonic sense of rotation (same as the planetary rotation) and diverges away from the equator in anticyclonic vortices. The superposition of columnar and ageostrophic circulation implies a coherent negative flow helicity in the northern hemisphere and positive helicity in the southern hemisphere. This simple picture was first derived by Busse (1975) and has been used as basis for a conceptual dynamo model. Although the flow pattern in numerical dynamos is complex and time-dependent, basically it still conforms with this generic picture in most geodynamo models.

Several authors have analyzed their numerical solutions in order to understand the basic mechanism by which the magnetic field is maintained. In the tradition of mean-field dynamo theory it is studied how large-scale (e.g. axisymmetric) poloidal field is generated from large-scale toroidal field and vice versa. There is general agreement that the axial dipole field is generated from the axisymmetric toroidal field by an $\alpha$-effect associated with the helical flow in the convection columns. In contrast to the classical $\alpha$-effect by small helical turbulent eddies, this is a 'macroscopic', $\alpha$-effect because the convection columns are not very much thinner than the width of convecting shell, at least in the models. Kageyama and Sato (1997) have been the first to describe this effect in detail.

The mechanism for generating the axisymmetric toroidal field, in which often two bundles of opposite polarity near the equatorial plane are prominent, is less clear. Olson et al. (1999) demonstrate that it is generated from the axisymmetric poloidal field by a similar macroscopic $\alpha$-affect in their models, i.e. the dynamo is of the $\alpha^{2}$-type. Also Ishihara and Kida (2002) find that field line stretching associated with the helical flow in the convection columns is the source for intense magnetic bundles that contribute both to the axisymmetric poloidal and toroidal field. Schrinner et al. $(2005,2007)$ studied in more detail the induction process in simple dynamo models in the framework of the mean-field concept. Their results confirm basically an $\alpha^{2}$-mechanism. However, a description by a simple isotropic $\alpha$-term is 
not adequate, and an $\alpha$-tensor with significant off-diagonal components and complex spatial variation is required.

Other authors present evidence for a significant role of the $\Omega$-effect in their models. Simitev and Busse (2005) find that the toroidal field in their models is mainly generated by the interaction of the axisymmetric toroidal flow field with the axisymmetric poloidal magnetic field, even though differential rotation is not a prominent flow component. Buffett and Bloxham (2002) show that in the model of Kuang and Bloxham (1999) the strong toroidal field energy is replenished by conversion of the kinetic energy of the axisymmetric toroidal flow. While in weakly driven numerical dynamo models the regions inside the tangent cylinder (north and south of the inner core) are quiescent, vigorous flow is found in more strongly driven models (Glatzmaier and Roberts 1995; Christensen et al. 1999). In these cases a strong axisymmetric toroidal field is often generated inside the tangent cylinder region by the shearing of poloidal field lines in a polar vortex whose sense of rotation changes from clockwise near the outer boundary to anticlockwise near the inner core. In conclusion, the overall role of the $\Omega$-effect in planetary dynamo models is not entirely clear. There may be genuine differences between models, some being of the $\alpha^{2}$-type and others of the $\alpha \Omega$-type.

\section{Comparison of Geodynamo Models with Earth's Field}

The following criteria can be applied to judge the similarity between the magnetic field of a dynamo model and the geomagnetic field: (1) agreement in dipole moment or generally in field strength, (2) agreement in the shape of the spatial power spectrum, (3) qualitative agreement in the magnetic field morphology at the core-mantle boundary, (4) agreement in the time scales of secular variation, (5) agreement in the frequency and characteristic properties of dipole reversals. Many published models satisfy some of these criteria and a few satisfy all of them to a fair degree. A good guide for a dynamo model to generate a closely Earth-like magnetic field is probably that the magnetic Reynolds number and the local Rossby number must assume the appropriate value. Other parameters may be less critical. We defer the discussion of the field strength of dynamo models to Sect. 8 and discuss the other criteria below.

The spectral power distribution as function of multipole degree $n$ is nearly white at the Earth's core-mantle boundary for $n>2$, at a level that is about a factor of ten below the dipole power (Fig. 3). Many geodynamo models in the dipolar regime reproduce it closely (see Christensen and Wicht 2007, for a more detailed discussion), although often the dipole is somewhat stronger or weaker relative to higher multipoles than in the observed spectrum (which represents basically a single snapshot in time). In addition, characteristic features in the morphology of the geomagnetic field at the core-mantle boundary are captured in a number of models. In Fig. 1 we compare the present geomagnetic field with a snapshot from a geodynamo model. In some models that have a large Ekman number and a fairly moderate value of the magnetic Reynolds number the model field can be compared directly. In a more advanced model, like the one shown here, the field must be low-pass filtered to the resolution that is available for the geomagnetic core field to reveal the similarity. The model reproduces the flux lobes at high latitudes, weak flux at the poles and it shows flux spots of both polarities at low latitudes.

The cause for these various magnetic structures in the core field has tentatively been inferred from the associated flow structures seen in the dynamo models (e.g. Christensen et al. 1998). The high-latitude flux concentrations are related to helical convection columns outside of the inner core tangent cylinder. Cyclonic vortices are associated with downwelling 
near the surface that concentrates magnetic flux. Low flux at the poles can be related to upwelling plumes near the rotation axis (Sreenivasan and Jones 2005). The plumes are accompanied by an anticyclonic vortex motion arising from a thermal wind effect. The variation of the geomagnetic field in the north polar region of the core-mantle boundary over the past hundred years, assuming that is to first order frozen into the fluid, indeed suggests that there is an anticlockwise motion inside the tangent cylinder (Olson and Aurnou 1999). Finally, bipolar pairs of flux spots at low latitudes are found in many dynamo models. They have been associated with the emergence of toroidal magnetic field tubes through the coremantle boundary, analogous to the mechanism for the formation of sunspots (Christensen et al. 1998; Christensen and Olson 2003). Sometimes the pairs are north-south rather than eastwest aligned and they show a polarity that is opposite to the general dipole polarity in their respective hemisphere, in particular in simpler dynamo models. Such configuration can arise because strong toroidal fields of opposite polarity are found at close distance near the equator and because the convective flow is strongly north-south aligned and acts on both toroidal tubes in a similar way. Comparable structures exist in the Earth's field at the core-mantle boundary (see Fig. 1a, below Africa and the Atlantic ocean) and have been explained by flux expulsion (Bloxham 1989). However, in the geomagnetic field they are more strongly offset from the equator than they are in dynamo models and other interpretations have been given for these field structures (Finlay and Jackson 2003).

Models that match Earth's magnetic Reynolds number show secular variations of the magnetic field of the right time scales. In fact, the fluid velocity in the core and hence the magnetic Reynolds number $R m$ can only be inferred from the secular variation of the geomagnetic field. Usually this is done assuming that the magnetic flux is frozen into the flow. Christensen and Tilgner (2004) have used results from numerical dynamo models to establish that the characteristic secular variation time scale, measured in terms of magnetic diffusion time, varies as $R m^{-1}$. With this scaling law they obtain a value around $R m=1000$ for the Earth, in fair agreement with estimates based on the frozen-flux assumption.

Geodynamo models that are in the right regime for dipole reversals (e.g. Glatzmaier and Roberts 1995; Glatzmaier et al. 1999; Kutzner and Christensen 2002; Takahashi et al. 2005; Wicht 2005) often show a degree of agreement with the paleomagnetic record that goes beyond the simple occurrence of reversals, even in cases with very modest parameters such as a relatively large values of the Ekman number. Figure 4 shows time series of the dipole tilt, dipole moment and relative dipole field strength in such a model. Traits that are similarly found in the geomagnetic field are: (1) The directional change of the dipole field is a relatively brief event, compared to the length of the period in which the dipole is nearly aligned with the rotation axis. (2) The dipole moment starts to drop before the directional change occurs. During the reversal the magnetic field is multipolar. (3) Aside from complete reversals, strong changes in the dipole direction that are brief and non-persistent also occur (geomagnetic excursions). The actual frequency of reversals in geodynamo models seems to depend on the fine tuning of parameters and is also controlled by second order effects, such as the non-uniform pattern of heat flow at the core-mantle boundary that is imposed by a heterogeneous structure of Earth's lower mantle (Glatzmaier et al. 1999).

\section{Scaling of Dynamo Properties}

An important question is what controls the vigor of convection and the strength of the magnetic field in a planetary dynamo. Some heuristic scaling laws based on mixing length theory or an assumed magnetostrophic force balance have been proposed for relating the velocity 


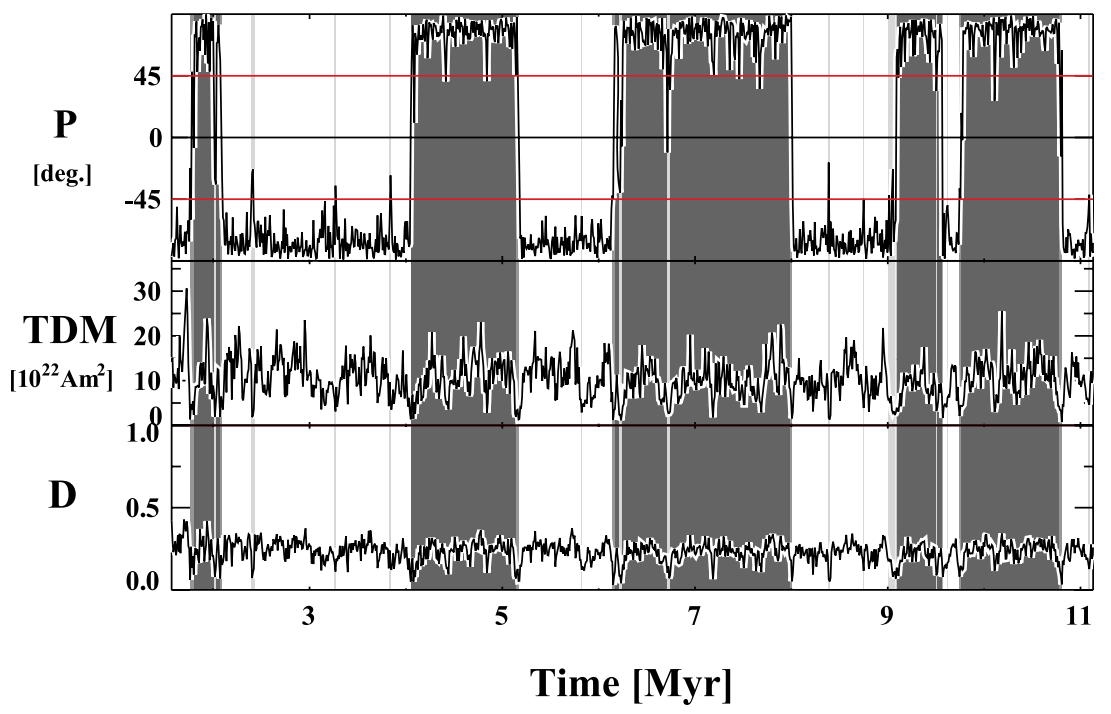

Fig. 4 Time series of the dipole tilt with respect to the equator $(\mathrm{P})$, the true dipole moment (TDM), and the dipole strength relative to the total field strength at the core mantle boundary (D) for a dynamo model with $E=10^{-3}, R^{*}=0.5, P m=10, P r=1$. The present TDM of the geomagnetic field is $8 \times 10^{22} \mathrm{Am}^{2}$. Dark and light bands indicate polarity intervals (unpublished, courtesy of Johannes Wicht)

to the heat flux, or more generally, to the buoyancy flux, which also comprises compositionally driven convection (Stevenson 1979, 2003; Starchenko and Jones 2002). These theories predict that the velocity should scale with the buoyancy flux to the $1 / 3$ or $1 / 2$ power, respectively. The usual assumption on the magnetic field strength for a dynamo in a magnetostrophic force balance is that the Elsasser number (1), representing the ratio of Lorentz forces to Coriolis forces, must be of order one inside the dynamo region, which sets the value of $B$.

The availability of a sufficiently large set of dynamo solutions which cover a decent range of control parameter space has led Christensen and Tilgner (2004) and Christensen and Aubert (2006) to derive scaling laws in a partly empirical way from numerical model results. Since the model values of several control parameters are far removed from planetary values, the question arises if the models are in the same dynamical regime. In particular, viscous friction which is thought to be negligible in planetary cores, has been suspected to play a major role in the models.

Christensen and Aubert (2006) tested if the values of viscous, thermal and magnetic diffusion constants influence the first-order properties of the numerical dynamos. In terms of non-dimensional parameters, the role of diffusion is controlled by the Ekman number and the two Prandtl numbers. Taking the modified form of the Rayleigh number (9) rather than the conventional Rayleigh number as the fourth control parameter has the advantage that it is independent of any of the diffusion constants. Because for planetary dynamos the driving heat flux or buoyancy flux is more readily estimated than the superadiabatic temperature contrast, Christensen and Aubert (2006) use a flux-based modified Rayleigh number

$$
R a_{Q}^{*}=\frac{r_{i}}{r_{c}} \frac{q_{\text {buoy }}}{\Omega^{3} D^{2}},
$$


where $q_{\text {buoy }}$ is the convective buoyancy flux (per unit area on the outer boundary) and $r_{i}$ the radius of the inner core. The total flux $Q_{\text {buoy }}$ as a function of radius is constant in their models. For purely thermal convection $q_{\text {buoy }}=\alpha g_{o} q_{\text {conv }} /\left(\rho c_{p}\right)$, where $q_{\text {conv }}$ is the convected heat flux and $c_{p}$ the heat capacity. $R a_{Q}^{*}$ is a non-dimensional measure for the power generated by buoyancy forces. The characteric flow velocity, magnetic field strength and heat transport properties of the dynamo solutions are expressed by non-dimensional numbers that are defined such that they do not relate to any of the diffusion parameters. These are the Rossby number

$$
R o=\frac{U}{\Omega D},
$$

the Lorentz number

$$
L o=\frac{B}{\sqrt{\mu \rho} \Omega D},
$$

and a modified Nusselt number

$$
N u^{*}=\frac{r_{i}}{r_{c}} \frac{q_{c o n v}}{\rho c_{p} \Delta T \Omega D},
$$

where $U$ and $B$ refer to the mean (rms) values of velocity and magnetic field inside the dynamo. Covering a range of at least two orders of magnitude in each of the parameters describing diffusive processes and six orders of magnitude for $R a_{Q}^{*}$, Christensen and Aubert (2006) found that the results depend at most weakly on $E, P r$ and $P m$. The three characteristic numbers $\mathrm{Nu}^{*}, \mathrm{Ro}$ and $L o$ relate by simple power-laws to the modified Rayleigh number $R a_{Q}^{*}$, with exponents of approximately $1 / 2,2 / 5$ and $1 / 3$, respectively. The exponent for the Rossby number is intermediate between the value derived from mixing length theory and that from magnetostrophic balance. It can be rationalized by assuming a triple balance of buoyancy, inertial forces and Coriolis forces (Aubert et al. 2001). The exponent for the Lorentz number can be understood on the basis that ohmic dissipation must be balanced by the power generated by buoyancy and that the ratio of magnetic energy to ohmic dissipation (the ohmic dissipation time constant) is inversely related to the magnetic Reynolds number (Christensen and Tilgner 2004). The fit to the model data could actually be improved by correcting for the energy lost by viscous dissipation. Figure 5 shows an updated plot of the corrected Lorentz number versus $R a_{Q}^{*}$. It is encouraging that all results can be collapsed fairly well on a simple dependence on the modified Rayleigh number, irrespective of the values of the three control parameters $E, P m, P r$ that refer to diffusive processes.

The Elsasser number, based on the rms field strength in the dynamo, was found to cover values between 0.1 and 100 in the various numerical models. Unless one is willing to accept that an 'order-one value', can actually lie within a range of three orders of magnitude, the Elsasser number rule does not seem to apply for the numerical dynamos, and may not be a generally valid guide to planetary magnetic field strength.

Casting the scaling law for the magnetic field into dimensional form and setting the exponent to $1 / 3$, the magnetic field strength is given by

$$
B \propto \mu_{o}^{1 / 2} \rho^{1 / 6} q_{\text {buoy }}^{1 / 3},
$$

and is independent of the rotation rate and of the conductivity. In that respect it differs from all previously suggested scaling laws. Of course, the conductivity must be high enough so that the critical magnetic Reynolds number for a dynamo is exceeded. Furthermore, rotation must be fast (the local Rossby number small) to obtain a dipolar dynamo. However, once 
Fig. 5 Lorentz number corrected for dissipation versus modified flux-based Rayleigh number. Model data are mostly from Christensen and Aubert (2006) with some additions. Shading of the symbols indicates the value of the magnetic Prandtl number, where darker means a lower value. Crosses inside the main symbol indicate $\operatorname{Pr}>1$ and circles indicate $P r<1$. The line represents the best fit for a forced slope of $1 / 3 . f_{\text {ohm }}$ is the contribution of ohmic dissipation to total dissipation. The shaded region for the geodynamo is based on estimates for the core field strength and buoyancy flux assuming $f_{\text {ohm }} \approx 1$ in the core

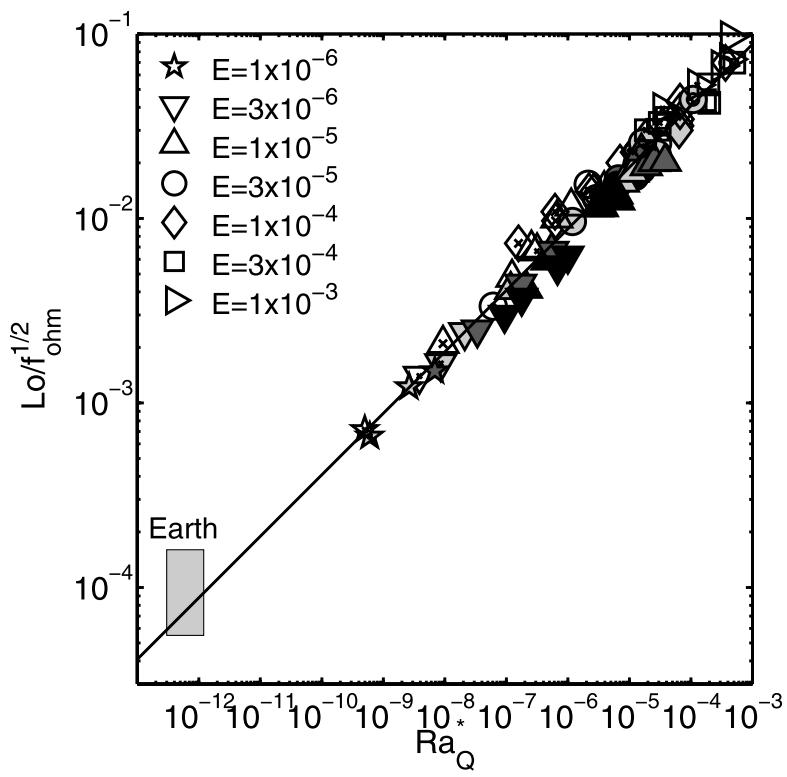

these conditions are met, (16) implies that the precise values of the conductivity and of the rotation rate have no influence on the magnetic field strength.

For testing whether the scaling laws are compliant with the geodynamo, the modified Rayleigh number and the Lorentz number of the Earth's core must be determined. Estimates for the buoyancy flux in the Earth's core suffer from substantial uncertainties and rely on indirect arguments. Using their scaling law that relates velocity to the buoyancy flux, Christensen and Aubert (2006) derived a value $Q_{\text {buoy }}=4 \pi r_{c}^{2} q_{\text {buoy }} \approx 3 \times 10^{4} \mathrm{~kg} \mathrm{~s}^{-1}$ for a typical flow velocity of $1 \mathrm{~mm} / \mathrm{s}$ obtained from secular variation. This value for the buoyancy flux is rather low and implies that the heat flow at the core-mantle boundary is close to the conductive heat flow along an adiabatic gradient. Most other estimates for the core heat flow are higher (e.g. Nimmo 2007). Considering a range of $3-12 \times 10^{4} \mathrm{~kg} \mathrm{~s}^{-1}$, the modified Rayleigh number for Earth's core is of the order $10^{-12}$. To estimate the mean field strength inside the core requires an assumption of how this relates to the 'observed', strength at the top of the core, which is $0.26 \mathrm{mT}$ for the dipole part of the field. Taking their dynamo models with an Earth-like field morphology as a guide, in which the toroidal field and the poloidal field are of similar strength, Christensen and Aubert (2006) estimated a ratio of 6-7 between the mean internal field strength and the dipole strength at the core surface. Considering values between 1 and $3 \mathrm{mT}$ for the core field, the Lorentz number is in the range $0.6-1.6 \times 10^{-4}$.

Even though the scaling law must be extrapolated a long way from the model parameter values to Earth values, the estimates for the geodynamo fall on the line defined by the various model results (Fig. 5). This strongly suggests that despite viscosity is far too large, the model dynamos operate in the same dynamical regime as the geodynamo does and that the agreement, for example in magnetic field morphology and reversal behavior, is not only a coincidence.

Christensen and Aubert (2006) applied their scaling law also to Jupiter, where the buoyancy flux can be estimated from the planet's excess luminosity. They predict a field strength of $8 \mathrm{mT}$ inside the dynamo. This is in good agreement with the observed dipole moment, assuming a similar ratio between mean internal field strength and dipole strength as in case of 
Fig. 6 Lorentz number based on the observed dipole moments normalized by estimated values of the modified flux-based Rayleigh number plotted against the local Rossby number for the magnetic planets in the solar system. Shaded ranges indicate predicted values based on numerical model results in the dipolar regime (left) and the multipolar regime (right)

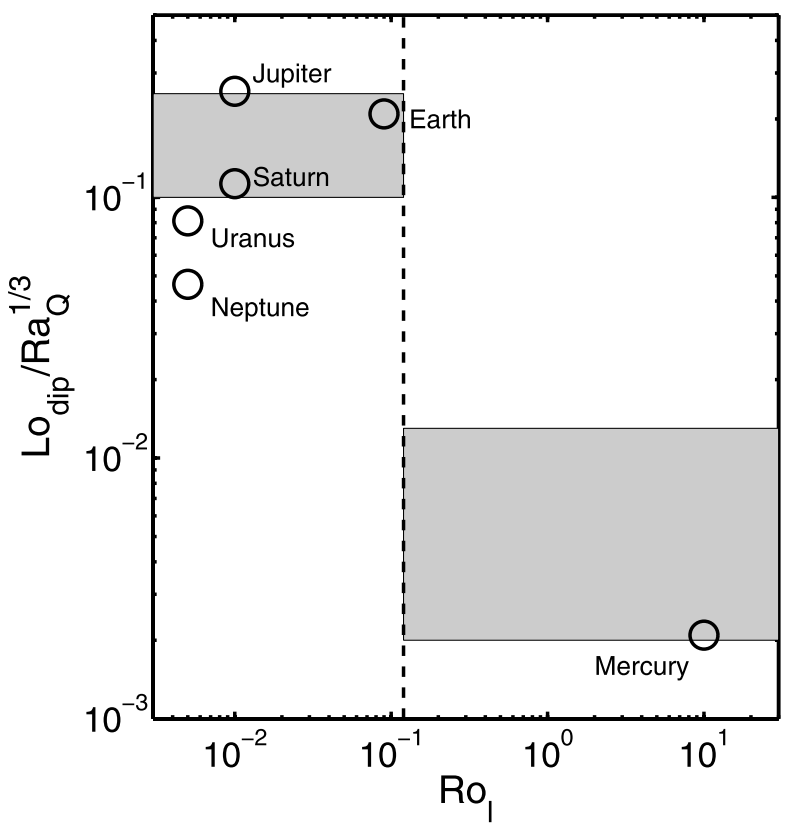

the Earth. Despite a much larger buoyancy flux, the bigger size and the more rapid rotation put Jupiter's dynamo at lower values of $R a_{Q}^{*}$ and $L o$ compared to the geodynamo.

Olson and Christensen (2006) used an even larger set of numerical dynamos, many of them taken from the literature, to derive a scaling law for the dipole moment as the most fundamental observable property of planetary magnetic fields. Expressing the dipole field strength again by a Lorentz number $L o_{\text {dip }}$, they confirm that it depends on the cubic root of the power driving the dynamo (expressed by $R a_{Q}^{*}$ ) as long as the magnetic field is dominated by the dipole. The scatter is somewhat larger than in the case of scaling the mean internal field, partly because a more heterogeneous set of dynamo models with different boundary conditions has been considered, and partly because the dipole is just one component of the field that does not need to keep a constant ratio to the total field when parameters are changed. The normalized dipole Lorentz number $L o_{\text {dip }} / R a_{Q}^{* 1 / 3}$ is nearly constant in the dipolar regime, but drops by more than an order of magnitude upon transition to the non-dipolar regime at values of the local Rossby number around 0.12 .

Applying their scaling law that relates the local Rossby number to the control parameters, Olson and Christensen (2006) find that all planets except Mercury should fall into the dipolar regime $\left(R o_{\ell}<0.12\right)$. The scaling law for $L o_{d i p}$ then predicts dipole moments in fair agreement with their observed values for most planets, despite large uncertainties in some cases on their internal properties and particularly the buoyancy flux (Fig. 6).

\section{Specific Models for Various Planets Other than Earth}

In order to explain idiosyncrasies in the structure or strength of the magnetic field of various planets dedicated dynamo models have been presented in recent years. Several of them rely on the existence of stably stratified layers in the fluid core of the planet. 
Fig. 7 Radial magnetic field in a model for Mercury's dynamo with a partly stable core.

Parameters are $E=10^{-4}$, $R a_{Q}^{*}=6 \times 10^{-4}, P m=3$, $\operatorname{Pr}=1$, inner core radius half the core radius and unstable layer thickness $44 \%$ of fluid core thickness. Top panel: at the top of the dynamo region (deep inside the core) with color contour step 100,000 nT; bottom panel: at Mercury's surface with step $100 \mathrm{nT}$
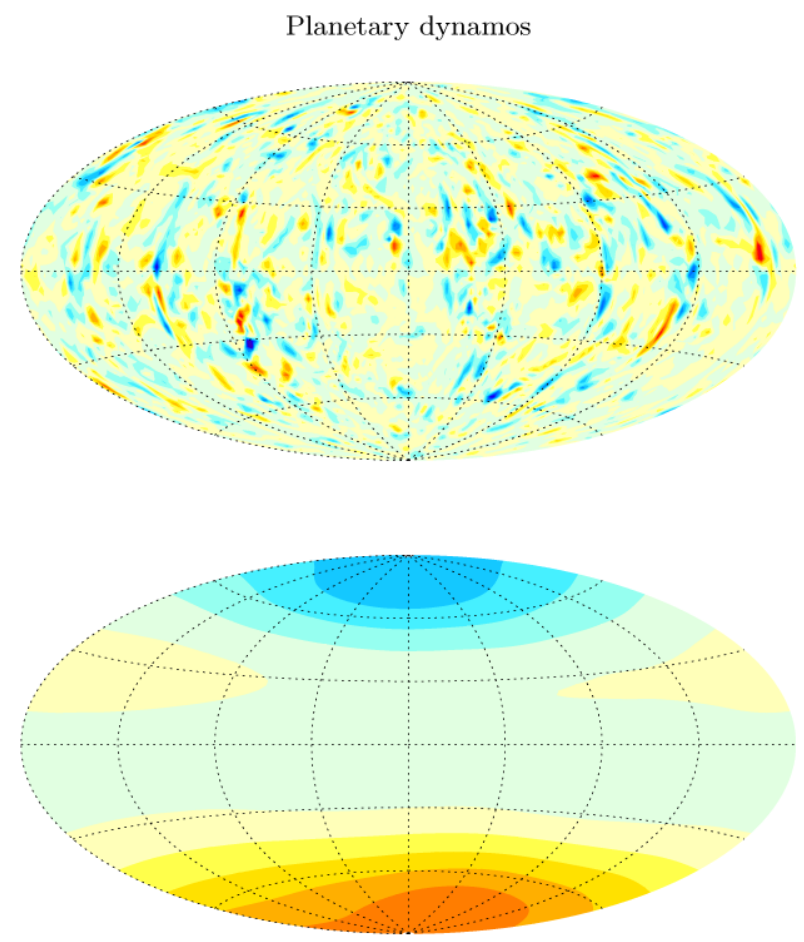

\subsection{Mercury}

The main problem with Mercury's magnetic field is to reconcile its relative weakness with the assumption of a hydromagnetic dynamo operating in the large iron core of the planet, whose outer boundary is at approximately 0.75 planetary radii. Observations of Mercury's forced libration (Margot et al. 2007) strongly indicate that Mercury's core is at least partially liquid. The existence of a solid inner core is likely, but its size is unconstrained. Dynamo models with a very large inner core (Stanley et al. 2005; Takahashi and Matsushima 2006) or with a very small inner core (Heimpel et al. 2005) succeeded in producing relatively weak magnetic fields in the exterior. However, the field in these models is either still too strong by a factor of ten or more, or it contains strong higher multipole components. Magnetometer data from the recent Messenger flyby have reinforced the preliminary conclusion from Mariner 10 data that the internal field is large-scaled and dominated by a slightly tilted dipole (Anderson et al. 2008). This, however, is in conflict with the prediction that Mercury should be in the multipolar dynamo regime based on a large value of the local Rossby number caused by the planet's very slow rotation (Fig. 6).

Christensen (2006) and Christensen and Wicht (2008) present dynamo models in which only a deep sublayer of the fluid core is convecting, whereas the upper region is stably stratified. This is based on thermal evolution models that predict a heat flow at Mercury's core-mantle boundary substantially less than the heat that can be conducted along an adiabatic temperature gradient (Breuer et al. 2007). Compositional buoyancy and the latent heat of inner core growth make the deep core region convectively unstable. Here a strong magnetic field is generated, which is small-scaled in models where the local Rossby number exceeds the threshold value for the multipolar regime (Fig. 7). The small-scale field varies 
rapidly with time. Therefore, it is strongly attenuated by a skin effect in the conducting stable layer and is virtually unobservable outside the core. The dipole component makes only a small contribution inside the dynamo, but varies more slowly. Hence it can penetrate through the stable layer and dominates the structure of the very weak field at the planetary surface (Fig. 7, bottom; note the factor 1000 difference in the color scheme).

\subsection{Saturn}

Stevenson $(1980,1982)$ suggested that stable stratification at the top of Saturn's metallic hydrogen layer could be the cause for the extremely high degree of axisymmetry in the planet's magnetic field. In Saturn the stratification can arise because helium may be partly immiscible with metallic hydrogen near the top of the metallic layer. While the density stratification suppresses convection, it allows for toroidal flow, in particular differential rotation. Let us assume for simplicity that the whole stable layer rotates like a uniform shell with respect to the underlying dynamo region and that the dynamo field is stationary. Seen from a reference frame that is fixed to the rotating shell, the non-axisymmetric field components will become time-dependent, whereas the axisymmetric components remain stationary. If the magnetic Reynolds number characterizing the shell motion is large enough, a skin effect will eliminate the non-axisymmetric parts of the field, leaving the axisymmetric components unaffected.

Christensen and Wicht (2008) find in their models (originally intended for Mercury's dynamo) that latitudinal differences in the heat flow from the dynamo region into the stable shell drive strong differential rotation as a thermal wind circulation. In their models the magnetic field inside the dynamo region has strong non-axisymmetric contributions, whereas the field outside the core has a high degree of axisymmetry. The latter disappears when in a control experiment the differential rotation in the stable layers is suppressed. In model cases where the local Rossby number is below the threshold value for the dipole-multipole transition, which should be the case in Saturn (see Fig. 6), the axial dipole is a strong and slowly time-varying component of the magnetic field inside the dynamo. Consequently the field outside the core is much stronger than it is in the case of Mercury. Although the dipolar models in Christensen and Wicht (2008) have not been tuned to Saturn parameters, they produce axisymmetric magnetic fields of a similar strength as Saturn's field and basically support Stevenson's hypothesis for the cause of axisymmetry.

\subsection{Uranus and Neptune}

The observed fields of Uranus and Neptune are multipolar. The rule of the local Rossby number would put them into the dipolar regime (Fig. 6), and may fail in these cases. Stanley and Bloxham $(2004,2006)$ present a dynamo model with a thin convecting shell that surrounds a fluid conducting but stable core region. Such a structure had been proposed to explain the relatively low excess luminosity of the planets. Some of their dynamo models generate magnetic fields that agree well with the observed distribution of power in the dipole, quadrupole and octupole components.

The conductivity of the ionic liquid in Uranus and Neptune is lower than that of metallic liquids by a factor of $10^{2}-10^{3}$. Gómez-Pérez and Heimpel (2007) showed in dynamo models that the magnetic field becomes less dipolar when the magnetic diffusivity is increased relative to viscosity. 


\section{Discussion}

Numerical dynamo models based on the direct numerical simulation of the fundamental MHD equations are remarkably successful in matching the main properties of the geomagnetic field and to some extent those of other planetary magnetic fields. In this respect modeling of planetary dynamos seems to be more advanced than modeling the solar dynamo. The reasons for the success of planetary dynamo models are a matter of speculation, but the following points may be important:

(1) Density stratification (compressibility) plays a small role in planetary dynamos, which at least eases the task. (2) It is possible to fully resolve the magnetic field structure and therefore the details of the magnetic induction process. Put differently, direct numerical simulations at the correct value of the magnetic Reynolds number are possible. (3) Although the model viscosity and thermal diffusivity are far larger than realistic microscopic values, the scaling laws obtained from systematic parameter studies suggest that they are low enough to not play a first-order role. (4) It seems that the large-scale flow structure, which is responsible for magnetic induction, is modeled realistically. Small flow scales may be important in planetary cores through their feedback on the large-scale flow. These small scales are missing in the models, but their effect is perhaps similar to that obtained by the interaction of the smallest resolved scales and the large scales in the models when the local Rossby number has the appropriate value. As in the case of the magnetic Reynolds number, simulations at Earth-like values of $R o_{\ell}$ are numerically feasible.

Acknowledgements We thank Johannes Wicht for providing Fig. 4. This research was supported in part by National Science Foundation (NSF) under Grant No. NSF PHY05-51164. KITP preprint number NSFKITP-08-104. NSF also sponsors the National Center for Atmospheric Research.

\section{References}

B.J. Anderson, M.H. Acuña, H. Korth et al., Science 321, 82 (2008)

J. Aubert, D. Brito, H.C. Nataf, P. Cardin, J.P. Masson, Phys. Earth Planet. Int. 128, 51 (2001)

J. Bloxham, Philos. Trans. R. Soc. Lond. 87, 669 (1989)

D. Breuer, S.A. Hauck II, M. Buske, M. Pauer, T. Spohn, Space Sci. Rev. 229-260, 132 (2007)

A.S. Brun, M.S. Miesch, J. Toomre, Astrophys. J. 614, 1073 (2004)

B.A. Buffett, J. Bloxham, Geophys. J. Int. 149, 211 (2002)

F.H. Busse, Geophys. J. R. Astron. Soc. 42, 437 (1975)

P. Caligari, F. Moreno-Insertis, M. Schüssler, Astrophys. J. 441, 886 (1995)

P. Charbonneau, Living Rev. Sol. Phys. (2005). http://www.livingreviews.org/lrsp-2005-2

U.R. Christensen, J. Fluid Mech. 470, 115 (2002)

U.R. Christensen, Nature 444, 1056 (2006)

U.R. Christensen, J. Aubert, Geophys. J. Int. 166, 97 (2006)

U.R. Christensen, P. Olson, Phys. Earth Planet. Int. 138, 39 (2003)

U.R. Christensen, P. Olson, G.A. Glatzmaier, Geophys. Res. Lett. 25, 1565 (1998)

U.R. Christensen, P. Olson, G.A. Glatzmaier, Geophys. J. Int. 138, 393 (1999)

U.R. Christensen, A. Tilgner, Nature 429, 169 (2004)

U.R. Christensen, J. Wicht, in Core Dynamics, ed. by G. Schubert. Treatise of Geophysics, vol. 8 (Elsevier, Amsterdam, 2007), pp. 245-282

U.R. Christensen, J. Wicht, Icarus 196, 16 (2008)

V. Courtillot, P. Olson, Earth Planet. Sci. Lett. 260, 495 (2007)

Y. Fan, Living Rev. Sol. Phys. (2004). http://www.livingreviews.org/lrsp-2004-1

C.C. Finlay, A. Jackson, Science 300, 2084 (2003)

G.A. Glatzmaier, R.S. Coe, L. Hongre, P.H. Roberts, Nature 401, 885 (1999)

G.A. Glatzmaier, P.H. Roberts, Nature 337, 203 (1995)

G.A. Glatzmaier, P.H. Roberts, Physica D 97, 81 (1996)

G.A. Glatzmaier, P.H. Roberts, Contemp. Phys. 38, 269 (1997) 
N. Gómez-Pérez, M. Heimpel, Geophys. Astrophys. Fluid Dyn. 101, 371 (2007)

E. Grote, F.H. Busse, Phys. Rev. 62, 4457 (2000)

E. Grote, F.H. Busse, A. Tilgner, Phys. Earth Planet. Int. 117, 259 (2000)

D. Gubbins, J. Bloxham, Geophys. J. R. Astron. Soc. 80, 695 (1985)

M. Heimpel, J.M. Aurnou, F.M. Al-Shamali, N. Gómez-Pérez, Earth Planet. Sci. Lett. 236, 542 (2005)

D.W. Hughes, R. Rosner, N.O. Weiss, The Solar Tachocline (Cambridge University Press, Cambridge, 2007)

N. Ishihara, S. Kida, J. Fluid Mech. 465, 1 (2002)

A. Jackson, A.R.T. Jonkers, M.R. Walker, Philos. Trans. R. Soc. Lond. A 358, 957 (2000)

A. Kageyama, T. Sato, Phys. Rev. E 55, 4617 (1997)

M. Korte, C.G. Constable, Geochem. Geophys. Geosyst. 6, Q02H16 (2005)

F. Krause, K.-H. Rädler, Mean-Field Magnetohydrodynamics and Dynamo Theory (Pergamon Press, Oxford, 1980)

W. Kuang, J. Bloxham, Nature 389, 371 (1997)

W. Kuang, J. Bloxham, J. Comput. Phys. 153, 51 (1999)

C. Kutzner, U.R. Christensen, Phys. Earth Planet. Int. 131, 29 (2002)

C. Kutzner, U.R. Christensen, Geophys. J. Int. 157, 1105 (2004)

J.L. Margot, S.J. Peale, M.A. Slade, I.V. Holin, Science 316, 710 (2007)

S. Maus, M. Rother, C. Stolle et al., Geochem. Geophys. Geosyst. 7, Q07008 (2006)

M.S. Miesch, Living Rev. Sol. Phys. (2005). http://www.livingreviews.org/lrsp-2005-1

F. Nimmo, in Core Dynamics, ed. by G. Schubert. Treatise of Geophysics, vol. 8 (Elsevier, Amsterdam, 2007), pp. 31-65

N. Olsen, G. Hulot, T.J. Sabaja, in Geomagnetism, ed. by G. Schubert. Treatise of Geophysics, vol. 5 (Elsevier, Amsterdam, 2007), pp. 33-75

P. Olson, J. Aurnou, Nature 402, 170 (1999)

P. Olson, U.R. Christensen, Earth Planet. Sci. Lett. 250, 561 (2006)

P. Olson, U.R. Christensen, G.A. Glatzmaier, J. Geophys. Res. 104, 10,383 (1999)

M. Ossendrijver, Astron. Astrophys. Rev. 11, 287 (2003)

V. Rama Murthy, W. van Westrenen, Y. Fei, Nature 423, 163 (2003)

P.H. Roberts, in Geomagnetism, vol. 2, ed. by J. Jacobs (Academic Press, London, 1987), pp. 251-306

M. Schrinner, K.-H. Rädler, D. Schmitt, M. Rheinhardt, U. Christensen, Astron. Nachr. 326, 245 (2005)

M. Schrinner, K.-H. Rädler, D. Schmitt, M. Rheinhardt, U. Christensen, Geophys. Astrophys. Fluid Dyn. 101, 81 (2007)

R. Simitev, F.H. Busse, J. Fluid Mech. 532, 365 (2005)

B. Sreenivasan, C.A. Jones, Geophys. Res. Lett. 32, L20301 (2005)

B. Sreenivasan, C.A. Jones, Geophys. J. Int. 164, 467 (2006)

S. Stanley, J. Bloxham, Nature 428, 151 (2004)

S. Stanley, J. Bloxham, Icarus 184, 556 (2006)

S. Stanley, J. Bloxham, W.E. Hutchison, Earth Planet. Sci. Lett. 234, 341 (2005)

S.V. Starchenko, C.A. Jones, Icarus 157, 426 (2002)

D.J. Stevenson, Geophys. Astrophys. Fluid Dyn. 12, 139 (1979)

D.J. Stevenson, Science 208, 746 (1980)

D.J. Stevenson, Geophys. Astrophys. Fluid Dyn. 21, 113 (1982)

D.J. Stevenson, Earth Planet. Sci. Lett. 208, 1 (2003)

F. Takahashi, M. Matsushima, Geophys. Res. Lett. 33, L10202 (2006)

F. Takahashi, M. Matsushima, Y. Honkura, Science 309, 459 (2005)

J.A. Tarduno, R.D. Cottrell, M.K. Watkeys, D. Bauch, Nature 466, 657 (2007)

J. Wicht, Geophys. J. Int. 162, 371 (2005) 